\title{
Clinical Course of Steroid Sensitive Nephrotic Syndrome in Children: Outcome and Outlook
}

\author{
Svitlana Fomina ${ }^{*}, 1$, Tatjana Pavlenko*,2, Erling Englund ${ }^{3}$ and Ingretta Bagdasarova ${ }^{1}$ \\ ${ }^{I}$ Department of Pediatrics, State Institution "Institute of Nephrology of the Academy of Medical Science of Ukraine", \\ Kyiv, Ukraine \\ ${ }^{2}$ Department of Mathematics, Royal Institute of Technology, SE-100 44 Stockholm, Sweden \\ ${ }^{3}$ Västernorrland County Council, Research and Development Centre, Sundsvall, Sweden
}

\begin{abstract}
Introduction: The aim of our study was to investigate the relative efficiency and adverse effects of various treatments of steroid sensitive nephrotic syndrome (SSNS) in children, and to determine factors associated with relapse risk in these patients.

Materials and Method: We retrospectively studied the data from 690 SSNS children treated in referral center over 25 years. The analyzed treatment protocols were: Prednisolone (PRED, eight weeks in a dose $1.5-2.0 \mathrm{mg} / \mathrm{kg}$, then it tapering and given for 9-12 months), Chlorambucil (CHL, cumulative dose 28.5-30 mg/kg), Cyclophosphamide intravenously (CYC I.V., cumulative dose of 30-36 mg/kg, then supporting dose of CHL, cumulative dose of 20-25 mg/kg) and intramuscular (CYC I.M., cumulative dose of $120-150 \mathrm{mg} / \mathrm{kg}$ ). The alkylating agents were used after remission induction by PRED and under its protection.

Results: Cumulative relapse-free survival was $81.9 \%, 69.0 \%$ and $64.5 \%$ after 12,36 and 60 months, respectively. In multivariate analyses, relapse risk was associated with age of treatment ( $<6$ years), and both PRED and CYC I.V. The only predictive factor for early relapse was PRED, unlike two and more relapses group where PRED and CYC I.V. as well as age from 3 to 6 years was highly prognostic. The high probability of sustained remission in combination with relatively mild adverse effects was observed for PRED used at first episode and CHL used at relapse.
\end{abstract}

Conclusion: To summarize, our protocols characterized by the prolonged PRED and CHL demonstrated promising results and should be considered as an efficient alternative strategy in SSNS management.

Keywords: Nephrotic syndrome, steroid sensitive, cyclophosphamide, chlorambucil, relapse intensity, relapse-free survival, predictive factors.

\section{INTRODUCTION}

Childhood nephrotic syndrome (NS) is often steroid sensitive (SS), usually with favorable long-term prognosis characterized however by a relapsing course in $50-80 \%$ of cases [1-5]. The relapsing patients come to be re-treated with prednisolone (PRED) which results in a high incidence of adverse effects (AE). Various treatment options include alternative agents for steroid-sparing effects such as alkylating agents, antimetabolites or calcineurin inhibitors. However, it is difficult to predict the non-relapsing course of SSNS, and remission rates after three years was have been reported as being between $24 \%$ and $45 \%$ [3-5].

The goals of our study were to investigate the relative efficiency of the various treatment protocols in childhood SSNS, to estimate the risk of relapse during 60 months follow up, to specify the predictive variables for relapsing

*Address correspondence to these authors at the State Institution "Institute of Nephrology of the Academy of Medical Science of Ukraine", Department of Pediatrics, 17b Degtyarivska str, Kiev, Ukraine; Tel: int +46-8 790 8466, Fax: int+46-8 723 1788; E-mail: sfomina@meta.ua

Department of Mathematics, Royal Institute of Technology, SE-100 44 Stockholm, Sweden; E-mail: pavlenko@math.kth.se course of the disease, and to access the treatment-related AEs.

\section{METHODS}

\section{Patients}

In the present retrospective study we reviewed the outcomes of 690 SSNS children treated in the referral clinic between January 1980 and May 2006. The model of NS management and treatment protocols were legislatively approved by the Ministry of Public Health of Ukraine.

The initial activity of the disease was diagnosed on the basis of classic signs: nephrotic-range proteinuria $(>3 \mathrm{~g} / 24$ hours, in the youngest age group $-1 \mathrm{~g} / \mathrm{m}^{2}$ ), serum albumin $<25 \mathrm{~g} / \mathrm{L}$, serum cholesterol $>6.5 \mathrm{mmol} / \mathrm{L}$, and edema. SS was defined as a complete remission (clinical and laboratory data) after six weeks of PRED (dose of $1.5-2 \mathrm{mg} / \mathrm{kg} / \mathrm{day}$ ). Relapse was defined as the recurrence of nephrotic range proteinuria and serum abnormality after the urine had been protein free of at least four weeks. Frequent relapsing clinical course was defined as three or more relapses during consequent 6 months.

The children were followed up in the clinic from 1.5 years and up to the age of 18 years, and if the disease was 
still active, they were transferred to an adult department. The following data were recorded: age of onset, duration of the disease, the presence of hematuria or hypertension, initial renal insufficiency, the number of relapses before the beginning of the treatment in the clinic, and type of treatment regimens in clinic. We also examined laboratory tests such as serum creatinine, serum albumin, serum cholesterol, glomerular filtration rate, urinalysis, and 24-h urinary protein excretion. Hematuria was defined as $>5 \times 10^{6}$ red blood cells/L in uncentrifuged urine. Hypertension was defined as blood pressure (systolic and/or diastolic) $>95^{\text {th }}$ percentile for age, gender, height percentile. Initial renal insufficiency was defined as a reversible increase of the serum creatinine level (100-460 $\mu \mathrm{mol} / \mathrm{l})$ accompanied by a decrease of the glomerular filtration rate according to the Schwartz formula in the initial observation of less then three months [6].

The patients were followed up once a week in the first three months, every three months in the first year, and every six months thereafter.

Children with age of NS onset $<1.5$ years, follow-up after immunosuppressant treatment less then twelve months and those with secondary NS, were excluded from this study. Furthermore, only those patients who indicated the sustained remission at the moment of the treatment withdrawal were included (0 time point in all graphs). Steroid dependent SSNS (episodes with relapse during the tapering of PRED or within two weeks of ending treatment) were also excluded from this study.

The Ethics Committee of the State Institution "Institute of Nephrology of the Academy Medical Science of Ukraine" approved of this study.

\section{Treatment Protocol}

The national treatment protocols were used thought the study, see [6].

PRED as the initial treatment was prescribed for eight weeks in dose $1.5-2.0 \mathrm{mg} / \mathrm{kg}$ body weight up to a maximum of $60 \mathrm{mg} /$ day, divided into two or three doses. Then it was decreased by $5 \mathrm{mg} /$ day to the supporting dose (30-50\% of initial dose) which was given on alternate-day or intermittent mode (three times a week) for 9-12 months with tapered by $2.5 \mathrm{mg}$ every eight weeks till $2.5-5 \mathrm{mg}$. In a case of any intercurrent concomitant disease under the supporting treatment, the dose of PRED was temporary increased up to $15-20 \mathrm{mg}$ /day during 7-14 days for relapse prevention.

In relapse, an immunosuppressive agent was applied after six weeks of PRED under transition to the supporting PRED therapy. First line steroid-sparing treatment was an alkylating agent Chlorambucil (CHL) or Cyclophosphamide (CYC) which was used during the eight weeks in the maximum dose. CHL was administrated in dose $0.2 \mathrm{mg} / \mathrm{kg} /$ day orally (cumulative dose of $8.5-10 \mathrm{mg} / \mathrm{kg}$ ). CYC was mostly given to patients with long NS history. It was initiated at a pulse dose intravenously (I.V.) of $10-25 \mathrm{mg} / \mathrm{kg}$ once in three weeks No. 3 ( $\leq 1 \mathrm{~g} /$ injection, cumulative dose of $30-36 \mathrm{mg} / \mathrm{kg}$ ) or every third day intramuscular (I.M.) in dose $6-9 \mathrm{mg} / \mathrm{kg} /$ injection (cumulative dose of $120-150 \mathrm{mg} / \mathrm{kg}$ ). CYC I.M. was given before the year 1995 (domestic based protocol) but CYC I.V. was used regular from the year 1995. After initial (maximum) dose, both CHL and CYC I.V. were transferred to the supporting dose of CHL $(0.1 \mathrm{mg} / \mathrm{kg} / \mathrm{day})$ which were then received during 6-9 months (cumulative dose of $20-25 \mathrm{mg} / \mathrm{kg}$ ), simultaneously with PRED.

\section{Statistical Analysis}

The statistical analyses were performed using STATISTICA 10.0 for Windows Version (Statsoft, Inc. 1984-2011) and IBM SPSS 19.0. Data not normally distributed were summarized as percentages, medians and ranges. Comparisons between groups were performed using Mann-Whitney and proportion tests. The cumulative frequency chart and the relapse rate per patient were used to illustrate the treatment efficiency. Kaplan-Meier survival technique was used to illustrate the relapse rates during 60months follow-up. The comparison of survival rates was performed using the log-rank test for two samples and an extension of Gehan's generalized Wilcoxon test and Peto and Peto's generalized Wilcoxon tests for multiple comparisons. The Cox proportional hazards regression was applied to identify a set of explanatory variables with high predictive potential and explore the simultaneous effects of several variables on the risk of NS relapsing course. Multinomial (logistic) regression was used to identify the risk factors of developing the highly relapsing course of NS. AEs of the treatment were measured as a relative frequency of event per patient from the cohort.

\section{A p-value of less than 0.05 was defined as significant.}

\section{RESULTS}

There were 447 boys and 243 girls included in the study (sex ratio 1.8). Their ages ranged from 1 to 17 years at the disease onset (median 5).

The entire cohort of children was stratified at the first step into the following age groups: from 1.5 to $<3$ years (1), from three to $<6$ years (2), from six to $<9$ years (3), from nine to $<12$ years, and from twelve to 18 years. However, the corresponding Kaplan-Meier curves for the two oldest groups revealed identical behavior of the relapse rate and were therefore merged together (from nine to 18 years), resulting in four age groups for the subsequent analyses.

The median duration of NS before beginning the treatment protocol in our clinic was 12 months (1-120) with overall relapse rate before admission 0.79. Previous PRED treatment courses have been given in local clinic in $475 / 68.8 \%$ cases. The follow up period in the clinic was ranged from 12 to 288 months (median 64).

The percentage of patients in complete remission was $75.1 \%, 46.4 \%$ and $31.7 \%$ after 12,36 and 60 months, respectively. The cumulative relapse free survival at the same time pointed as $81.9 \%, 69.0 \%$ and $64.5 \%$, respectively, after Kaplan-Meier estimators corrected for censoring $(47 / 6.8 \%$ was lost to follow up before 12 month, further $119 / 17.2 \%$ of patients was lost up to 36 month, and $81 / 11.7 \%$ more up 60 months).

The estimated probability of the sustained remission up to 60 months analyzed in the different age groups is illustrated in Fig. (1), and a highly significant difference in the event rate depending on the age was discovered. Younger ages were relapsed more intensively and the probability of NS recurrence in the late follow-up was $45.7 \%$ for age group from 1.5 to $<3$ 
years, $39.4 \%$ - from three to $<6$ years, $26.8 \%$ - from six to $<9$ years and $17.7 \%$ for age group from nine to 18 years, respectively $\left(\chi^{2}=28.0, \mathrm{p}<0.001\right)$. The remission rates were estimated as complements to the Kaplan-Meier probabilities, resulted in $49.2 \%, 57.5 \%, 70.1 \%$, and $79.3 \%$, respectively, for the four age groups. It is important to note that in the case of relapse, the duration of remission was similar in all age groups (median 12 months, ranged from 1 to 132).
The significant difference was discovered in the rate of relapse after treatment in our clinic between NS children with the previous PRED history and those treated firstly (Fig. 2). The cumulative probability of having a relapse free clinical period up to 60 month was $72.1 \%$ and $47.6 \%$ for the group with and without a long time history of the NS, respectively $(\log -$ rank $=-7.50, \mathrm{p}<0.001)$.

To analyze the effect of our treatment protocols, the duration of the remission was plotted as the cumulative

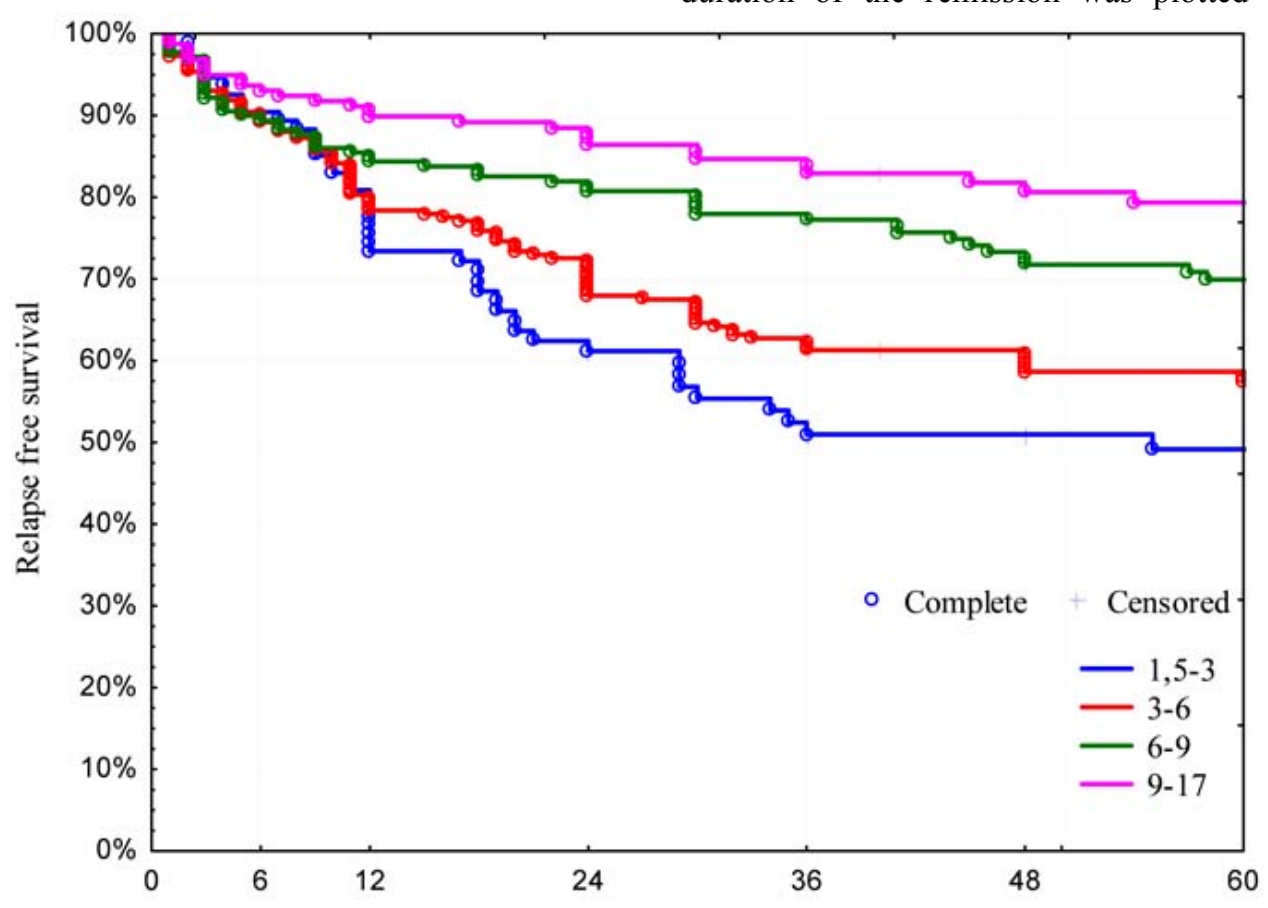

Time since treatment withdrawal (months)

Fig. (1). Kaplan-Meier survival curve for estimated probability of the sustained remission up to 60 month in the analyzed age groups: from 1.5 to $<3$ years $(n=94)$, from three to $<6$ years $(n=259)$, from six to $<9$ years $(n=179)$, from nine to 18 years $(n=158)$; total number -690 , complete - 235/34.1\%, censored - 455/65.9\%.

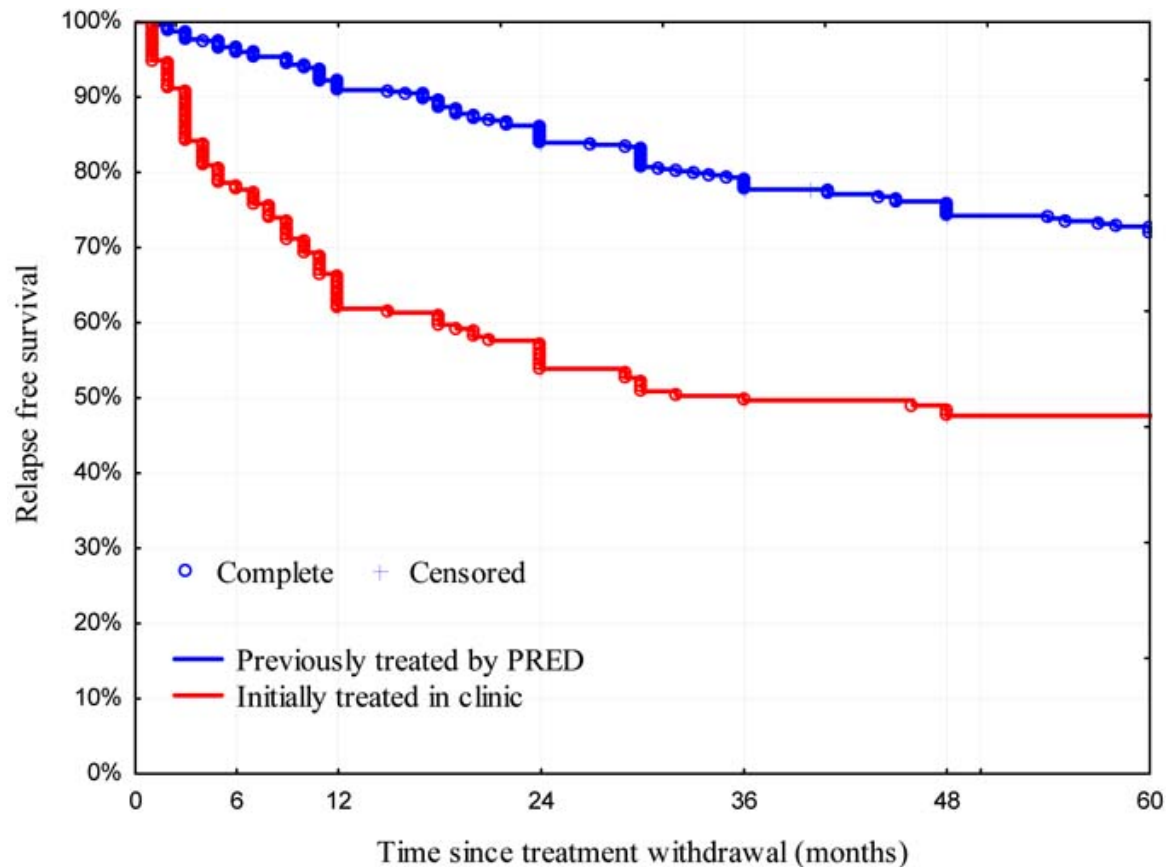

Fig. (2). Kaplan-Meier survival curve for estimated probability of the sustained remission up to 60 month in SSNS children previously treated by PRED and non treated disease history: total number - 475 and 221, complete $-114 / 24.0 \%$ and 107/49.8\%, censored - 361/76.0\% and 108/50.2\%, respectively (SSNS steroid sensitive nephrotic syndrome, PRED Prednisolone). 


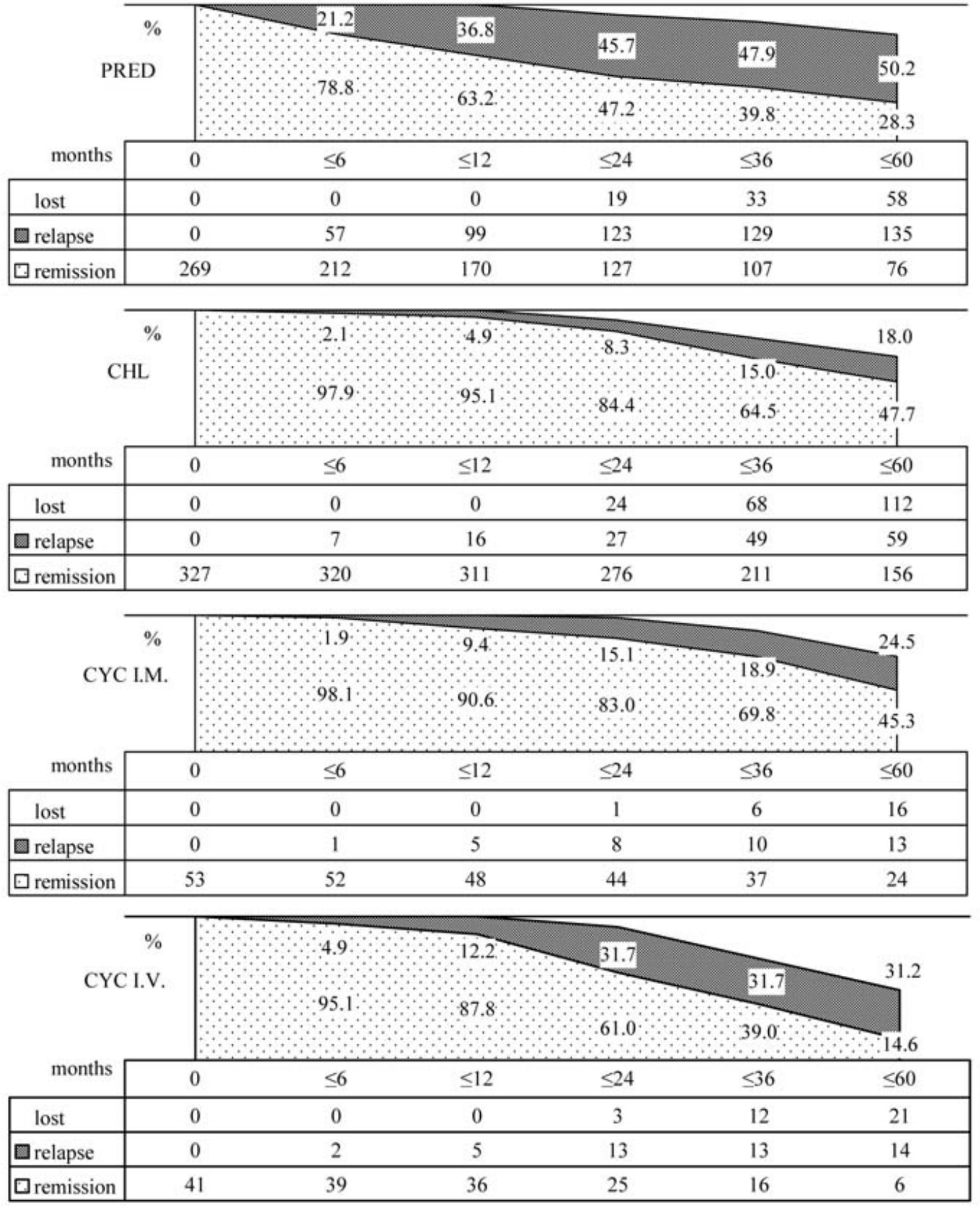

Fig. (3). Cumulative frequency charts of the remission and relapse rate for various treatment strategies: PRED, CHL, CYC I.M. and CYC I.V. (PRED Prednisolone protocol, CHL Chlorambucil protocol, CYC I.M. Cyclophosphamide intramuscular protocol, CYC I.V. Cyclophosphamide intravenously protocol)

frequency chart over time of observation for PRED alone $(\mathrm{n}=269)$, PRED combined with CHL (specified as "CHL", $\mathrm{n}=327$ ), CYC with PRED ("CYC" both I.V. and I.M., n=41 and $n=53$, respectively). More stable remission was observed in groups treated by CHL and CYC I.M. (Fig. 3).
Most of patients in the PRED group exhibited the first relapse until $12^{\text {th }}$ month of follow up being relapsed in every second case to the end of observation time. The CYC I.V. was more efficient in retaining the remission (time to relapse 18 months, range 2-48), than PRED, being however less 


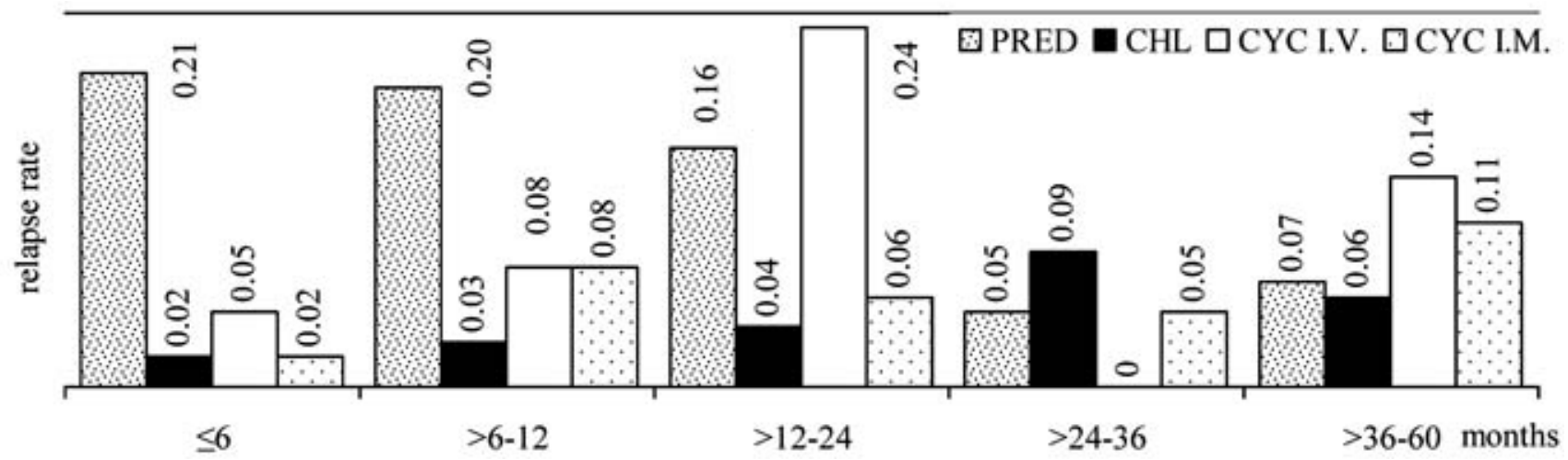

Fig. (4). The relapse rate per patient for various treatment protocols with SSNS children (SSNS steroid sensitive nephrotic syndrome, $P R E D$ Prednisolone protocol, CHL Chlorambucil protocol, CYC I.M. Cyclophosphamide intramuscular protocol, CYC I.V. Cyclophosphamide intravenously protocol).

effective than other protocols with alkylating agents: 26 months (range 5-60) for CYC I.M. and 36 months (range 1132) for CHL, respectively.

To analyze the treatment efficiency, the relapse rate per patient was estimated for a specific time interval. This rate was defined as the number of patients with relapse divided by the number of patients accumulated, proportionally to the time in the interval in which they were followed up (Fig. 4). PRED being used along gave ten times higher relapse rate during the first six months than CHL and CYC I.M. protocols. It remained high until 24 months and started to decrease in the last two time interval. The rate patterns in the both treatments CHL and CYC I.M. were similar: low relapse rates at the earlier observation times, and tendency to increase it in the late follow up. The highest relapse rate in CYC I.V. was observed from $12^{\text {th }}$ to $24^{\text {th }}$ month.

The overall picture of the treatment efficiency is presented as the percentage of the relapse free patients distributed over the treatments and follow up months (Table 1). Highly significant variation in the cumulative relapse free survival rates for the treatment studied was found ( $<0.001$ by Gehan's test) with most pronounced difference between PRED and CHL.

Table 1. Cumulative Relapse-Free Survival Rate (\%) for Different Treatment Protocols (PRED Prednisolone Protocol, CHL Chlorambucil Protocol, CYC I.M. Cyclophosphamide Intramuscular Protocol, CYC I.V. Cyclophosphamide Intravenously Protocol)

\begin{tabular}{|c|c|c|c|}
\hline \multirow{2}{*}{ Treatment Protocol } & \multicolumn{3}{|c|}{ Follow Up, Months } \\
\cline { 2 - 4 } & $\mathbf{1 2}$ & $\mathbf{3 6}$ & $\mathbf{6 0}$ \\
\hline \hline PRED & 63.4 & 50.2 & 47.2 \\
\hline CHL & 95.5 & 82.8 & 78.3 \\
\hline CYC I.V. & 87.9 & 66.7 & 58.1 \\
\hline CYC I.M. & 90.9 & 80.3 & 72.7 \\
\hline
\end{tabular}

When analyzing different treatment protocols for the frequently relapsing patients we found that more than two relapses up to 60 month were observed in 9/3.3\% PRED treated children and in $6 / 1.4 \%$ after alkylating agents therapy $(\mathrm{p}=0.053)$. Therapy with CHL and CYC I.M. seldom led to the frequently relapsing NS $(3 / 0.9 \%, \mathrm{p}=0.027$ compare to PRED, and $1 / 1.9 \%$ cases, $p=0.328$ compared to PRED, respectively), whereas the higher relapse rate was observed for CYC I.V. ( $2 / 4.9 \%, p=0.274$ compared to PRED).

A strong association between the time of the first relapse occurrence and the frequently relapsed course for PRED treatment was observed. Out of 9 patients with the frequently relapsed course, $5 / 55.6 \%$ had their first relapse during the first six months after PRED withdrawal, unlike the rarely relapsed ones (one or two relapses during the follow up) which get $52 / 40,6 \%$ early relapses out of $128(\mathrm{p}=0.185)$. The same pattern relating the early relapse and subsequent relapsing course was also observed in patients receiving the alkylating agents; out of 6 frequently relapsing NS there were $3 / 50.0 \%$, and out of 92 rarely relapsing cases there were $7 / 7.6 \%$ early ones, respectively $(\mathrm{p}=0.013)$.

To identify predictive factors of relapse up to 60 month on nephrotic children, the Cox regression model (Model 1) was fitted (Table 2). Three variables (age group, treatment before and the treatment protocol in clinic) appeared to be highly significant individual prognostic factors. Both age groups and type of treatment demonstrated highly significant predictive potential in the multivariate model.

Another Cox regression model, with the relapse risk during the first six months after the treatment withdrawal and the same set of explanatory variables, was fitted (Model 2). The variables, treatment before and PRED treatment at the clinic were detected as highly significant when using the univariate approach $(\mathrm{HR}=0.16,95 \%$ CI $0.10-0.28$, pvalue $<0.001$, and $\mathrm{HR}=10.81,95 \%$ CI 4.93-23.7, pvalue $<0.001$, respectively). Other types of treatment protocols, CHL, CYC I.V. and I.M., were not significant for developing the early relapse, unlike the previous model, where the follow-up time was extended to 60 months. Some tendency to higher risk of the early relapse was observed in patients treated by CYC I.V. (HR=2.33, 95\% CI 0.48-11.21), unlike patients treated by CYC I.M. where the risk was lower $(\mathrm{HR}=0.90,95 \%$ CI $0.11-7.10)$. Notice that in both cases, the reference group was CHL protocol. The age group also lost its significance in association with early relapse.

With the multivariate model (Model 2), the only PRED treatment has shown significant prognostic effect on the early relapse $(\mathrm{HR}=7.84,95 \%$ CI $2.95-20.84$, p-value $<0.001)$. 
Table 2. Cox Proportional Hazard Model for NS with Relapse - Model 1 (PRED Prednisolone Protocol, CHL Chlorambucil Protocol, CYC I.M. Cyclophosphamide Intramuscular Protocol, CYC I.V. Cyclophosphamide Intravenously Protocol)

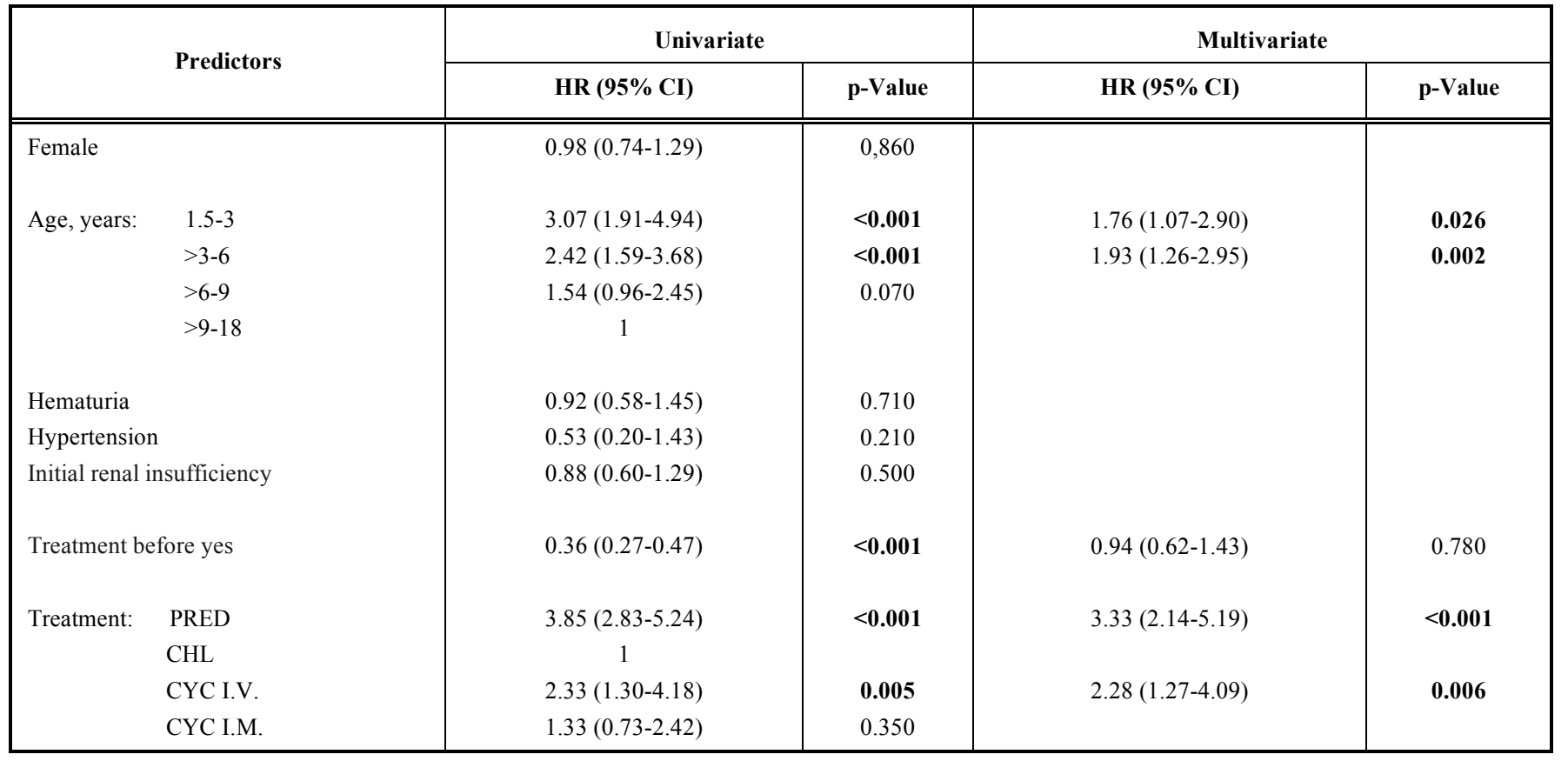

To determine risk factors related to the highly relapsing NS, the multinomial regression model was fitted (Table $\mathbf{3}$ ). The response variable was defined as a risk of developing one relapse in Model 3, and more than one relapse in Model 4 , in order to compare it with the relapse free course. In both univariate and multivariate approaches, the younger age and PRED treatment were significantly associated with the risk of getting one relapse compared to no relapse (Model 3). Having a treatment before reduced the risk of one relapse in the univariate model, but not in multivariate one. Similar patterns were also observed for the risk of developing two and more relapses: both the younger age and PRED treatment were highly significant predictors (Model 4). The multinomial regression model made it possible to capture the association between CYC treatment and developing highly relapsing NS course. The risk of more than one relapse was significantly elevated for CYC I.V. treated patients. Highly significant predictors of two and more relapses by multivariate analysis were age from three to six years and both treatment PRED and CYC I.V.

It has long been known, that relapsing clinical course requires repeated immunosuppressive therapy which in turn is related to the increase of the cumulative dosage and elevation of the risk of AEs. To study these effects of the treatments used, a random sample of medical records of 271 patients was taken. We considered the distribution of various types of AEs over the treatment strategies, which were presented in Table 4.

Most patients tolerated therapy well. Steroid-related AEs occurred in all the groups being less in CHL and CYC I.M. treated patients. Cushing features was mostly observed, followed by psychological disorders (hyperactivity, emotional instability, aggressive behavior). Transient glucosuria was also observed in all the groups but mainly in CHL. Leukemoid hematological reaction of myeloid type (myelocytes, metamyelocytes - young forms of leucocytes in peripheral blood sample) was documented in eleven patients. Five children developed severe AEs: osteoporosis was diagnosed in three cases and ulcerous illness in two ones. In total, 16 patients out of 271 had transient arterial hypertension. In a number of cases headache or rush was observed during the tapering PRED to supporting dose (Table 4).

As a result of total immune suppression various infections were observed with predominating in CYC-treated patients. The recurrence upper respiratory infections and pyogenic skin diseases occurred most frequently, whereas the pneumonia, urinary tract infections were limited. During these infections cytotoxic therapy was continued. Systemic infections were rare: one case of osteomyelitis and one case of sepsis were observed. In total, herpes zoster and chicken pox were experienced in 14 and 19 children, respectively. These types of AEs led to a therapeutic pause.

Hematological changes (anemia with $\mathrm{Hb}$ 86-104 g/L, leukopenia with WBC's count 2200-3400, thrombocytopenia with PLT $135-150 \times 10^{9} / \mathrm{L}$ ) were most frequently detected in alkylating agents' treated persons, especially CYC group, but they did not reach life-threatening levels.

Transitory cytolysis syndrome $(<5$ norm ALT) was observed in 44 patients (most of them were under CYC treatment) as hepatotoxicity, and it did not require alterations in the treatment protocols. As expected, at the alkylating agent treated patients observed hair loss, especially in CYC I.M. Eleven patients in the sample had transient intestinal syndrome as a frequent passage of loose stools during the first month of the treatment.

Endocrinological disorders were placed into a separate group since it was difficult to identify the real cause of their development. Overweight was observed in eleven patients, 
Table 3. Multinomial Logistic Regression Risk of One Relapse Compared to Non Relapse - Model 3, and Risk of Two and More Relapses Compared to Non Relapse - Model 4 (PRED Prednisolone Protocol, CHL Chlorambucil Protocol, CYC I.M. Cyclophosphamide Intramuscular Protocol, CYC I.V. Cyclophosphamide Intravenously Protocol)

\begin{tabular}{|c|c|c|c|c|c|}
\hline \multirow{2}{*}{\multicolumn{2}{|c|}{ Predictors }} & \multicolumn{2}{|c|}{ Univariate } & \multicolumn{2}{|c|}{ Multivariate } \\
\hline & & OR $(95 \% \mathrm{CI})$ & p-Value & OR $(95 \% \mathrm{CI})$ & p-Value \\
\hline \multicolumn{6}{|l|}{ Model 3} \\
\hline \multicolumn{2}{|l|}{ Female } & $0.98(0.68-1.39)$ & 0.890 & & \\
\hline \multicolumn{2}{|c|}{ Age, years: $1.5-3$} & $3.74(2.01-6.95)$ & $<0.001$ & $2.28(1.18-4.41)$ & 0.015 \\
\hline \multicolumn{2}{|c|}{$>3-6$} & $3.16(1.88-5.31)$ & $<0.001$ & $2.65(1.55-4.53)$ & $<0.001$ \\
\hline \multicolumn{2}{|c|}{$>6-9$} & $2.00(1.15-3.50)$ & 0.015 & $1.67(0.94-2.99)$ & 0.080 \\
\hline \multicolumn{2}{|c|}{$>9-18$} & 1 & & & \\
\hline \multicolumn{2}{|l|}{ Hematuria } & $0.89(0.50-1.57)$ & 0.680 & & \\
\hline \multicolumn{2}{|c|}{ Hypertension } & $0.53(0.18-1.60)$ & 0.260 & & \\
\hline \multicolumn{2}{|c|}{ Initial renal insufficiency } & $0.67(0.40-1.11)$ & 0.121 & & \\
\hline \multicolumn{2}{|c|}{ Treatment before yes } & $0.32(0.23-0.46)$ & $<0.001$ & $1.02(0.54-1.91)$ & 0.950 \\
\hline \multirow[t]{4}{*}{ Treatment: } & PRED & $3.81(2.60-5.58)$ & $<0.001$ & $3.44(1.85-6.42)$ & $<0.001$ \\
\hline & CHL & 1 & & & \\
\hline & CYC I.V. & $1.33(0.57-3.08)$ & 0.501 & & \\
\hline & CYC I.M. & $1.01(0.46-2.20)$ & 0.980 & & \\
\hline \multicolumn{6}{|l|}{ Model 4} \\
\hline \multicolumn{2}{|l|}{ Female } & $0.64(0.33-1.24)$ & 0.190 & & \\
\hline \multicolumn{2}{|c|}{ Age, years: $1.5-3$} & $3.79(1.29-11.20)$ & 0.016 & $2.85(0.92-8.87)$ & 0.070 \\
\hline \multicolumn{2}{|c|}{$>3-6$} & $3.75(1.50-9.40)$ & 0.005 & $3.34(1.31-8.51)$ & 0.011 \\
\hline \multicolumn{2}{|c|}{$>6-9$} & $1.37(0.46-4.05)$ & 0.570 & & \\
\hline \multicolumn{2}{|c|}{$>9-18$} & 1 & & & \\
\hline \multicolumn{2}{|l|}{ Hematuria } & $0.35(0.08-1.50)$ & 0.160 & & \\
\hline \multicolumn{6}{|c|}{ Hypertension* } \\
\hline \multicolumn{2}{|c|}{ Initial renal insufficiency } & $1.12(0.52-2.41)$ & 0.770 & & \\
\hline \multicolumn{2}{|c|}{ Treatment before yes } & $0.48(0.26-0.89)$ & 0.020 & $1.31(0.47-3.63)$ & 0.600 \\
\hline \multirow[t]{4}{*}{ Treatment: } & PRED & $3.46(1.74-6.88)$ & $<0.001$ & $3.68(1.32-10.28)$ & 0.013 \\
\hline & CHL & 1 & & & \\
\hline & CYC I.V. & $4.06(1.44-11.44)$ & 0.008 & $4.12(1.44-11.77)$ & 0.008 \\
\hline & CYC I.M. & $1.83(0.57-5.83)$ & 0.310 & & \\
\hline
\end{tabular}

*Due to no cases with "more than one relapse" in Hypertension group OR was not estimated.

and growth retardation was present in six children both with a long NS history. Hypohonadism was discovered in twelve cases but gonadotoxicity level was not detected due to ethical and social limitation. Out of 70 patients, 56 responded to the questionnaire at their adult age and reported about having at least one healthy child.

To date, there were not noted malignancies during follow up.

As expected, total number of AEs per patient was less in groups PRED (1.7). Besides, this index was not very high in CHL treated group (1.9), increased only in CYC I.V. (2.8), and in CYC I.M. (3.1).

\section{DISCUSSION}

The main goal of our study was to analyze the efficiency of the treatment strategies for SSNS used in the years 19802006 in Ukraine. Our results showed that PRED and cytostatic protocols applied at the clinic were in general consistent with the international approaches, however some specific features concerning doses and treatment duration still exist. One of such features is combined prolonged treatment with long term follow up. Even though over $30 \%$ of patients at the end of 60 months follow-up period were lost, one can claim that with high probability these patients were relapse free: our clinic is the main institute in Ukraine 
Table 4. AEs of the Treatment (n/\%) (PRED Prednisolone Protocol, CHL Chlorambucil Protocol, CYC I.M. Cyclophosphamide Intramuscular Protocol, CYC I.V. Cyclophosphamide Intravenously Protocol)

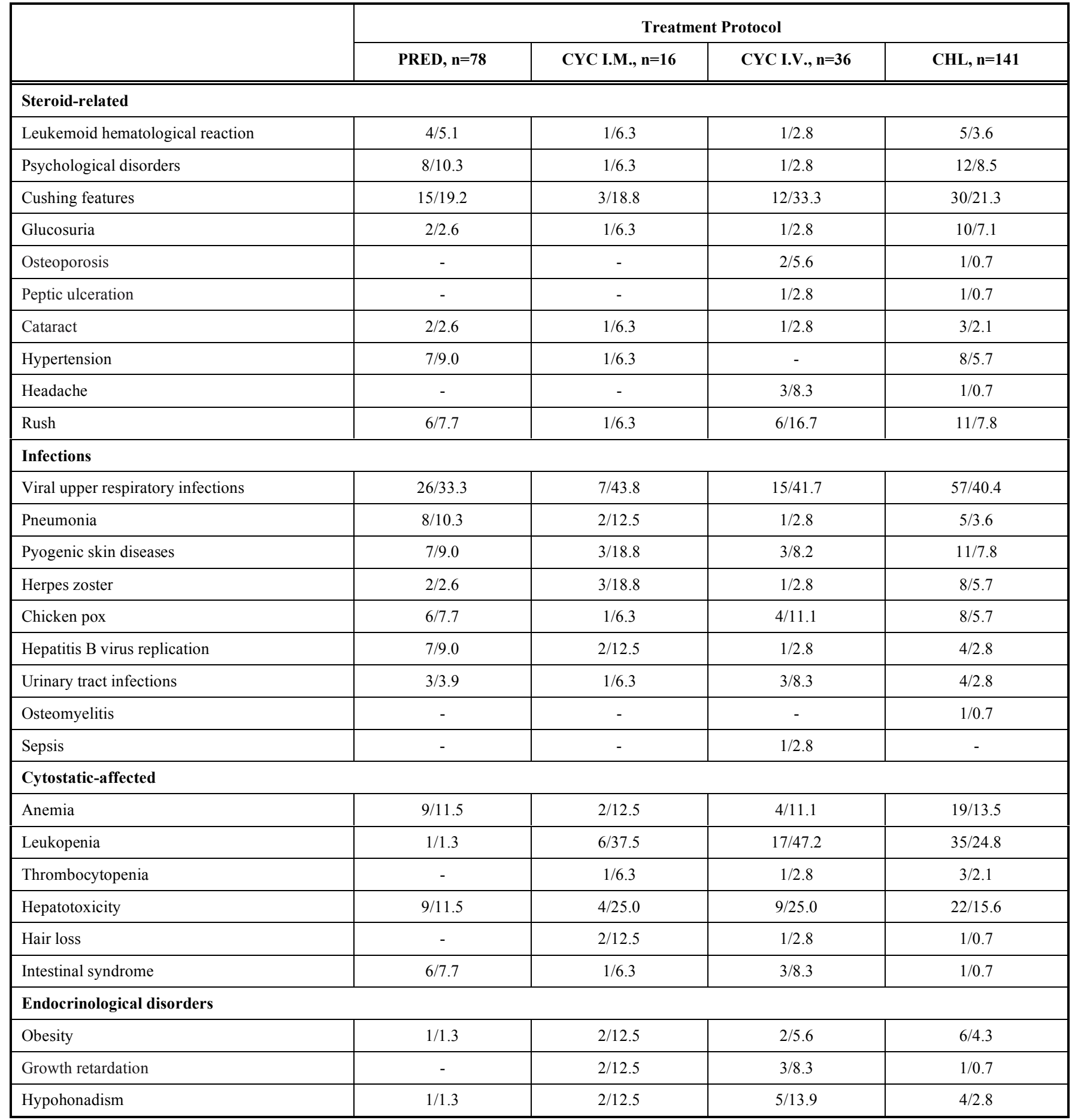

dealing with this type of disease and transfer of patients for the treatment abroad is almost unknown. Therefore these results can be extended to the whole cohort.

It has been shown that a number of factors related to patient age and treatment strategy has a high predictive potential for relapsing clinical course. In particular, the association between young age and recurrent SSNS has previously been reported by [3, 5, 7-9]. In our study, the two youngest ages (from 1.5 to $<3$ and from three to $<6$ years) were stably significant relapse prognostic variables in both
Cox and multinomial regression models. The elevated risk of the first relapse was observed in young ages, after at least six months of treatment withdrawal (Model 2), unlike other studies that did not report about the relationship between early relapse and age of patients [2]. Furthermore, the age from three to six years was highly related to the development of two and more relapses during 60 months (Model 3). In general, young age at onset and short initial remission were shown to be associated with frequent relapses. 
It has long been known that the patients with SSNS respond to PRED treatment alone, but majority of them have relapse and approximately $60-70 \%$ of children develop a steroid-dependent or frequently relapsing course; see e.g. [1, $3,5,10]$. At the same time, by prolonging PRED treatment the disease outcome usually becomes more favorable: the stable remission was observed in up $45-94 \%$ of cases during 24-36 months of follow-up [3, 10-14]. However, the longterm PRED treatment seriously increases the risk of AEs among these patients, which in turn motivates alteration of treatment. The steroid-sparing agents based treatment is the main approach for reducing relapse rate and thereby avoiding steroid-related AEs [3, 11, 14-16]. During the last years, the alkylating agents in the case of NS relapse are not of first priority. Nevertheless, CYC I.V. is usually recommended as an optional treatment to maintain remission in SSNS [7, 11, 13-22]. In different studies, the percentage of relapse-free patients varies between 5 and $67 \%$ during 24 months, and between 17 and $65 \%$ during 60 months, respectively $[5,18,19-24]$. To our best knowledge the data about applying CYC I.M. under the SSNS are not presented in the Western literature. The data concerning the use of CHL are nowadays rarely presented being usually reported in the earlier studies $[18,25,26]$.

Our study showed that the 12 months prolonged initial PRED treatment increased cumulative relapse-free survival up to $47.2 \%$ during 60 months after therapy withdrawal, which is better than the international results reported on shorter course of PRED. But the treatment efficiency analysis indicated that the PRED protocol (PRED along) led to disease recurrence primarily during the first 6 months after treatment withdrawal (Model 2), which is in agreement with the other studies, see e.g. $[9,27]$. Moreover, in the multivariate consideration, models fitted for the NS course prediction demonstrated that PRED treatment remained highly significant factor for all types of relapses analyzed (Models 1 to 4). This in part motivates the use of steroid sparring therapy already at the first SSNS relapse.

Another interesting finding of the study is that the previous PRED based treatment significantly contributed to reducing the relapse risk, including both early and frequently relapsing courses (Models 3 and 4).

As demonstrated in our analysis, the application of the alkylating agents has led to favorable clinical courses of SSNS; the similar results are also reported in [3, 14, 23, 28]. As usually recommended, first line CYC I.V. was applied during eight weeks and, unlike the international protocols, was then followed by supporting doses of CHL. This combined approach led to sustained remission in $58.1 \%$ during 60 months of follow up, which is comparable to the international studies [19, 21, 23]. But our analyses revealed that this treatment protocol had high predictive potential for two and more relapses in the last follow up (Model 4 and Fig. 4).

It was shown that CYC I.M. was more efficient than CYC I.V.; with CYC I.M. protocol, the relapse-free group was extended to $72.7 \%$ of cases after 60 months. It should be noted that the use of CYC I.M. was interrupted at our clinic at 1995, mostly due to elevated risk of development of AEs and treatment complications related to high cumulative doses. Nevertheless, we included our results on CYC I.M. in the study to have a more complete historical view. Since the results documented were positive, one can not exclude returning of the physician's interest to this regime of CYC.

The best results in SSNS treatment were achieved by applying the CHL protocol as a first alternative in relapse. It resulted in cumulative relapse free survival of $78.3 \%$ at the last follow up. Some earlier reports also suggested a superior effect of CHL over other alkylating agents $[18,25,26]$. But the straightforward comparison of the domestic and international treatment regimes is relative since in our patients the dosage of CHL was generally higher than standard one. Due to the high risk of complicated disease course, the application of CHL is restricted the international practice to some special cohorts of patients, and usually suggested as a second line therapy. However, during the last years, physicians return to the use of CHL in SSNS patients $[24,29]$. This motivates the consideration and analyses of our data on this treatment protocol.

It is well known that the number of AEs and their degree limited the duration of both PRED and alkylating agents' courses $[4,10,13,14,19,22]$; the general tendency is to reducing their cumulative dosage. However, though that our protocols provided relatively high drug cumulative dosages we did not observe the deterioration of AEs spectrum.

According to the previous studies, the percentage of steroid-dependent AEs was commonly high for both shot and long terms. Most often observed were Cushing features that were frequently recorded in children treated with international protocols (up to 66.6\%) $[10,13]$. The rate of this $\mathrm{AE}$ for our protocols did not exceed these bounds, ranging between 18.8 and $33.3 \%$.

The hypertension was reported in approximately 11$37.5 \%$ of patients in the international literature $[8,10,13$, 30 ] but only in $0-9.0 \%$ in our study. It is important that increase in blood pressure was detected after 4-5 weeks of treatment; it was controllable and limited in time to tapering PRED.

In our study, such short-time negative effects of PRED as hematological changes, glucosuria, rush and headache on treatment tapering, were considered to be significant for physical and psychological adaptation of patient. However, many other studies did not report such features. That might be either due to lucking of observations or due to ignoring such symptoms as having no importance. Another explanation could be that such features are related to the duration of our protocols.

Such serious AEs as cataract and osteoporosis were seen very rarely so that it was difficult to interpret them as a drug related. Unlike our approach, international studies reported these cases as steroid related AEs, even though they are also rarely seen $[4,8,10]$.

Infection is a well known concern in children with NS, especially when receiving both PRED and cytotoxic agents. Consistent with some previous reports, total infections level was moderate (up to $31.0-60.0 \%$ ) $[7,10,30]$. The dominating type of these AEs was viral upper respiratory infection. However, more serious AE problems in this study were caused by activation of Herpes, including chicken pox, especially under CYC treatment, sporadic sepsis and 
osteomyelitis, which led to discontinuation of cytotoxic therapy.

The hematological suppression with leukopenia was the most often observed and relatively complicated AEs under the alkylating agents treatment, which is reported by both our and international studies [3, 14, 20, 30]. This effect is usually considered as dose-dependent, and many therapeutic strategies strive to achieve the leukopenia in order to induce the long-term remission. Our results supported this approach, however without considering it as an ultimate intermediate goal of the treatment. Our strategy to reducing the risk of leucopenia was based on simultaneous administration of PRED and the alkylating agents [19].

Unlike the hematological changes, the long-term AEs of CHL or CYC are uncertain. It is well known that the prolonged or continuous use of alkylating agents is limited due to the risk of malignancy. Even though no neoplasia was found in our patients as $[2,21,23]$, there was no reliable data in the follow-up longer than 60 month.

It was also problematic to estimate the rate of infertility in the cohort under study. The negative effect of cytotoxic therapy is more pronounced for male than for female patients $[2-4,7,14,21]$. Due to relatively high dosage of alkylating agents in our protocols, we would expect elevation of the risk of infertility in long follow-up. However, since the birth rate is based not only on the potential biological ability for people to have children but also on their social behavior and habits, the detection of these problems had a high amount of subjectivity.

Other unfavorable consequences of the therapy, such as growth retardation, overweight or obesity, being causes by limitations of the prolonged using PRED and alkylating agents $[8,13]$, were minimized in the cohort under study, as it is reported in $[2,3,31]$. This in turn can be explained by reducing the number of re-treatments associated with reducing of total cumulative doses.

It is always extremely difficult to balance between the efficiency of NS therapy with stable remission and the risk of AEs. Furthermore, the accumulation of AEs related to previous treatments should also be taken into account for the risk evaluation in the group of retreated patients.

A special question in assessment of the treatment of SSNS in children is related to developing of relapsing or steroid-dependent NS course, which appeared in 30-60\% of patients [2-4]. In contrast, our treatment resulted in three or more relapses during 60 months follow up in $3.3 \%$ under PRED and in $4.9 \%$ cases under CYC I.V., respectively. This rate was further reduced under CHL and CYC I.M to $0.9 \%$ and $1.9 \%$, respectively.

When comparing the results of our study with others, certain limitations need to be acknowledged. Firstly, our patients were not randomized. Second, some children who had not relapsed and thus had not been seen at the clinic for 1.5 years or more have been omitted from the study. This might result in underestimation of relapse intensity for the whole cohort since our clinic is the leading in NS management in Ukraine. Third, due to long terms of our retrospective study and a large number of physicians participated, certain corrections might be done on the spread of their subjective evaluations of AEs in the medical records.

Finally, the reducing of the relapse rate during observational time, significantly contribute to improving of patients life quality, psychosocial adaptation and diminishing of the disease-dependent costs.

In conclusion, this study suggested that our protocols significantly reduced relapse intensity and increased the cumulative relapse-free survival. We found that prolongation of initial PRED treatment of SSNS had a beneficial effect on the subsequent clinical course of the disease. The modification of CHL used in our study was the most efficient protocol in patients with relapse of SSNS as a firstline choice. More studies are needed to further investigate the effects of PRED and CHL in childhood NS regarding the treatment protocols and AEs reduction.

\section{ACKNOWLEDGEMENTS}

The study was in part supported by the grant N00962/2008 from the Visby Program, Swedish Institute (http://www.si.se).

\section{CONFLICT OF INTEREST}

None declared.

\section{REFERENCES}

[1] International Study of Kidney Disease in Children. The primary nephrotic syndrome in children. Identification of patients with minimal change nephrotic syndrome from initial response to prednisolone. A report of the International Study of Kidney Disease in Children. J Pediatr 1981; 98: 561-4.

[2] Ruth E-M, Kemper MJ, Leumann EP, Laube GF, Neuhaus TJ. Children with steroid-sensitive nephrotic syndrome come of age: long-term outcome. J Pediatr 2005; 147: 202-7.

[3] Hodson EM, Willis NS, Craig JC. Non-corticosteroid treatment for nephrotic syndrome in children. Cochrane Database Syst Rev 2008; (1):CD002290 [updated 2008, cited 2010]. Available from: http://www.thecochranelibrary. com/

[4] Niaudet P. Long-term outcome of children with steroid-sensitive idiopathic nephrotic syndrome. Clin J Am Soc Nephrol 2009; 4: 1547-8.

[5] Esfahani ST, Madani A, Asgharian F, et al. Clinical course and outcome of children with steroid-sensitive nephrotic syndrome [serial on the Internet]. 2011 Feb; [cited 2011 March 12]; Pediatr Nephrol DOI 10.1007/s00467-011-1837-6. Available from: http:// www.springerlink.com/content/h603574m $7 \times 77185 \mathrm{~m} /$

[6] Fomina S, Pavlenko T, Englund E, Bagdasarova I. Clinical patterns and renal survival of nephrotic syndrome in childhood: a singlecenter study (1980-2006). Open Urol Nephrol J 2010; 3: 8-15.

[7] Latta K, von Schnakenburg C, Ehrich JHH. A meta-analysis of cytotoxic treatment for frequently relapsing nephrotic syndrome in children. Pediatr Nephrol 2001; 16: 271-82.

[8] Fakhouri F, Bocquet N, Taupin P, et al. Steroid-sensitive nephrotic syndrome: from childhood to adulthood. Am J Kidney Dis 2003; 41 (3): 550-7.

[9] Andersen RF, Thrane N, Noergaard K, Rytter L, Jespersen B, Rittig S. Early age at debut is a predictor of steroid-dependent and frequent relapsing nephrotic syndrome. Pediatr Nephrol 2010; 25: 1299-304.

[10] Hodson EM, Willis NS, Craig IC. Corticosteroid therapy for nephrotic syndrome in children. Cochrane Database Syst Rev 2007; (4): CD001533. [updated 2007, cited 2010]. Available from: http://www.thecochran elibrary. com/

[11] Arbeitsgemeinschaft fur Padiatrische Nephrologie. Cyclophosphamide treatment of steroid dependent nephrotic syndrome: comparison of 8 week with 12 week course. Arch Dis Child 1987; 62: 1102-06 
[12] Matsukura H, Inaba S, Shinozaki K, et al. Influence of prolonged corticosteroid therapy on the outcome of steroid-responsive nephrotic syndrome. Am J Nephrol 2001; 21 (5): 362-7.

[13] Lande MB, Gullion C, Hogg RJ, et al. Long versus standard initial steroid therapy for children with the nephrotic syndrome. A report from the Southwest Pediatric Nephrology Study Group. Pediatr Nephrol 2003; 18: 342-6.

[14] Bagga A. Management of steroid-sensitive nephritic syndrome: revised guidelines. Indian Pediatr 2008; 45: 203-14.

[15] Eddy AA, Symons JM. Nephrotic syndrome in childhood. Lancet 2003; 362 (9384): 629-39.

[16] Abeyagunawardena AS, Dillon MJ, Rees L, et al. The use of steroid-sparing agents in steroid-sensitive nephrotic syndrome. Pediatr Nephrol 2003; 18: 919-24.

[17] Hiraoka M, Tsukahara H, Matsubara K, et al. A randomized study of two long-course prednisolone regimens for nephrotic syndrome in children. Am J Kid Dis 2003; 41(6): 1155-62.

[18] Takeda A, Ohgushi H, Niimura F, Matsutani H. Long-term effects of immunosuppressants in steroid-dependent nephrotic syndrome. Pediatr Nephrol 1998; 12: 746-50.

[19] Bircan Z, Kara B. Intravenous cyclophosphamide is the drug of choice for steroid dependent nephrotic syndrome. Pediatr Int 2003; 45(1): 65-7.

[20] Vester U, Kranz B, Zimmermann S, et al. Cyclophosphamide in steroid-sensitive nephrotic syndrome: outcome and outlook. Pediatr Nephrol 2003; 18: 661-4.

[21] Azib S, Macher MA, Kwon T, et al. Cyclophosphamide in steroiddependent nephrotic syndrome [serial on the Internet]. $2011 \mathrm{Feb}$; [cited 2011 March 12]; Pediatr Nephrol DOI 10.1007/s00467-0111830-0. Available from: http://www.springerlink.com/content/g8 t7255q011hx528/

[22] Zagury A, de Oliveira AL, de Moraes CAP, et al. Long-term follow-up after cyclophosphamide therapy in steroid-dependent nephrotic syndrome [serial on the Internet]. $2011 \mathrm{Feb}$; [cited 2011 March 13]; Pediatr Nephrol DOI 10.1007/s00467-011-1825-x.
Available from: http://www.springerlink.com/content/c22j4717761 85774/

[23] Kyrieleis HA, Levtchenko EN., Wetzels JF. Long-term outcome after cyclophosphamide treatment in children with steroiddependent and frequently relapsing minimal change nephrotic syndrome. Am J Kidney Dis 2007; 49 (5): 592-7.

[24] Chen S-Y, Wu C-Y, Tsai I-J, Tsau Y-K. Treatment course of steroid-dependent nephrotic syndrome: Emphasized on treatment effect. Nephrology 2010; 15: 336-9.

[25] Arbeitsgemeinschaft fur Padiatrische Nephrologie. Effect of cytotoxic drugs in frequently relapsing nephritic with and without steroid dependece. N Engl J Med 1982; 306: 451-4.

[26] Niaudet P. Comparison of cyclosporin and chlorambucil in the treatment of steroid-dependent idiopathic nephrotic syndrome: a multicentre randomized controlled trial. The French Society of Paediatric Nephrology. Pediatr Nephrol 1992; 6 (1): 1-3.

[27] Yap H-K, Han EJS, Heng C-K, Gong W-K. Risk factors for steroid dependency in children with idiopathic nephrotic syndrome. Pediatr Nephrol 2001; 16: 1049-52.

[28] Van Husen M, Kemper MJ. New therapies in steroid-sensitive and steroid-resistant idiopathic nephrotic syndrome [serial on the Internet]. 2010 Nov; [cited 2011 January 11]; Pediatr Nephrol DOI 10.1007/s00467-010-1717-5. Available from: http://www.springer link.com/content/172603gk106483gg/

[29] Ueda N, Takahashi K, Akioka Y, Morooka M, Ogawa A, Tsuzuki $\mathrm{K}$. Beneficial effect of chlorambucil in steroid-dependent and cyclophosphamide-resistant minimal change nephrotic syndrome. J Nephrol 2009; 22 (5): 610-5.

[30] Donia AF, Ammar HM, El-Agroudy AE-B, Moustafa FE-H, Sobh MA-K. Long-term results of two unconventional agents in steroiddependent nephrotic children. Pediatr Nephrol 2005; 20: 1420-5.

[31] Simmonds J, Grundy N, Trompeter R, Tullus K. Long-term steroid treatment and growth: a study in steroid-dependent nephrotic syndrome. Arch Dis Child 2010; 95 (2): 146-9.

(C) Fomina et al.; Licensee Bentham Open.

This is an open access article licensed under the terms of the Creative Commons Attribution Non-Commercial License (http://creativecommons.org/licenses/by$\mathrm{nc} / 3.0 /$ ) which permits unrestricted, non-commercial use, distribution and reproduction in any medium, provided the work is properly cited. 